\title{
The challenges in paediatric pulmonary arterial hypertension
}

\author{
Maurice Beghetti ${ }^{1}$ and Rolf M.F. Berger ${ }^{2}$
}

Affiliations: 'Paediatric Cardiology Unit, Children's University Hospital Geneva, Geneva, Switzerland. ${ }^{2}$ Centre for Congenital Heart Diseases, Dept of Pediatric Cardiology, Beatrix Children's Hospital, University Medical Centre Groningen, University of Groningen, Groningen, The Netherlands.

Correspondence: Maurice Beghetti, Unité de cardiologie Pédiatrique, Hopital des Enfants, Cardiologie pédiatrique, Rue Willy-Donzé 6, 1205 Geneva, Switzerland. E-mail: maurice.beghettiahcuge.ch

ABSTRACT Pulmonary arterial hypertension $(\mathrm{PAH})$ is a rare, progressive disease affecting both adults and children. While overall survival has improved in recent years, the need for improved therapeutic approaches remains. Treatments for paediatric PAH have not yet been sufficiently examined, particularly regarding potential toxicities and optimal dosing, and there is a lack of appropriate clinical trial end-points and validated treatment goals that might enable a goal-oriented therapeutic approach. Adult randomised controlled trials in PAH are demonstrating a shift towards more long-term designs, focusing on mortality and morbidity end-points rather than changes in 6-min walking distance. However, such trial designs may not be feasible within the paediatric setting due to challenges such as sufficient recruitment and retention of paediatric patients. Consideration should, therefore, be given towards identifying optimal end-points for the paediatric population, allowing sufficient duration to evaluate efficacy and safety of potential treatments.

Herein we consider some of the complexities involved in the management of paediatric PAH, specifically presenting diagnostic challenges as well as reflecting on the lack of evidence currently available to support various therapeutic approaches within the paediatric population.

0 @ERSpublications

Paediatric pulmonary arterial hypertension: towards consistency in management and improved patient outcomes http://ow.ly/Cydpv

\section{Introduction}

Pulmonary arterial hypertension (PAH) is a rare disease of the cardiopulmonary system that can be diagnosed at any age. It is characterised by progressive proliferation and remodelling of the pulmonary vasculature, causing increased pulmonary vascular resistance (PVR) that ultimately leads to right ventricular failure [1]. Evidence for the epidemiology of PAH originates primarily from adult registries; however, several large, international registries have provided further understanding of the disease in the paediatric population [2-7].

Paediatric PAH is associated with a variety of underlying diseases and causes [5]. The disease is progressive, with the exception of persistent pulmonary hypertension in the newborn [8], spanning from infancy to adulthood and is associated with a poor prognosis; therefore, it is important for physicians to deliver the best possible care according to age for each patient.

Treatment of paediatric PAH is largely based on evidence from studies in adults and on the experience of clinicians in treating children. Diagnostic and therapeutic strategies for paediatric PAH have not been sufficiently examined, particularly with regard to potential toxicity and optimal dosing, and there is a lack of

Received: Aug 292014 | Accepted after revision: Oct 072014

Conflict of interest: Disclosures can be found alongside the online version of this article at err.ersjournals.com

Provenance: Publication of this peer-reviewed article was sponsored by Actelion Pharmaceuticals Ltd, Allschwil, Switzerland (principal sponsor, European Respiratory Review issue 134).

Copyright OERS 2014. ERR articles are open access and distributed under the terms of the Creative Commons Attribution Non-Commercial Licence 4.0. 
appropriate clinical trial end-points, hampering trials examining efficacy in children. Furthermore, there is a lack of appropriate treatment targets that would allow for goal-oriented treatment strategies in children. This review will focus on the challenges faced by physicians in paediatric $\mathrm{PAH}$, including the complexities of diagnosis and the limited evidence for effective PAH therapies in the paediatric population.

\section{Paediatric PAH}

Pulmonary hypertension characterisation and classification

Paediatric pulmonary hypertensive vascular disease is multifactorial and highly heterogeneous (fig. 1). While this disease shares some characteristics with $\mathrm{PAH}$ in adults, there are differences in associated conditions and comorbidities, co-existence of genetic or developmental disorders, and the broader context of growth and development (including underdevelopment and maladaptation). These differences suggest that the current classification, designed for adult PAH, does not suffice or requires the incorporation of dedicated paediatric aspects, particularly considering the effect such characteristics may have on diagnosis and treatment options for paediatric patients.

A Paediatric Taskforce of the Pulmonary Vascular Research Institute has proposed a diagnostic classification consisting of 10 main classes and 109 subclasses of this disorder, demonstrating its complex pathophysiology during growth [9].

With the aim to work on the creation of a common disease classification for both adults and children, a consensus from the 2013 World Symposium on Pulmonary Hypertension has updated the clinical classification of pulmonary hypertension and added several specific items related to paediatric pulmonary hypertension [10]. These include the addition of novel genetic disorders causing PAH, the repositioning of persistent pulmonary hypertension in the newborn as a separate subcategory within Group $1 \mathrm{PAH}$, the addition of congenital disorders involved in pulmonary hypertension due to left heart disease, and the emphasis of developmental lung diseases in Group $3 \mathrm{PAH}$, as a result of an increased understanding of lung structure and abnormal vascular growth in these disorders [10]. Currently, this classification has five main groups of pulmonary hypertension, each of which shares similar pathophysiological, histopathophysiological and clinical characteristics. While this approach falls short of an ideal classification for paediatric pulmonary hypertension, it has the advantages of: assisting consistent diagnosis and treatment; ensuring standardisation of registry data and improving trial conduct in homogeneous patient groups; and fostering improved communication amongst physicians, particularly during the transition from infancy to adulthood.

\section{PAH aetiology and survival}

The international TOPP (Tracking Outcomes and Practice in Pediatric Pulmonary Hypertension) registry shows that, of 362 children with PAH, 57\% had idiopathic PAH (IPAH) or familial PAH, a proportion similar to that observed in adult registries $[5,11,12]$. PAH associated with congenital heart disease (CHD) is relatively more frequent in children (36\%) than adults (10-11\%) [5], while PAH associated with connective tissue disease and other associated forms are very rare in children compared with the adult population $[4,5,13]$.

FIGURE 1 Multifactorial causes of paediatric pulmonary hypertensive vascular disease. Reproduced from [9] with permission from the publisher.

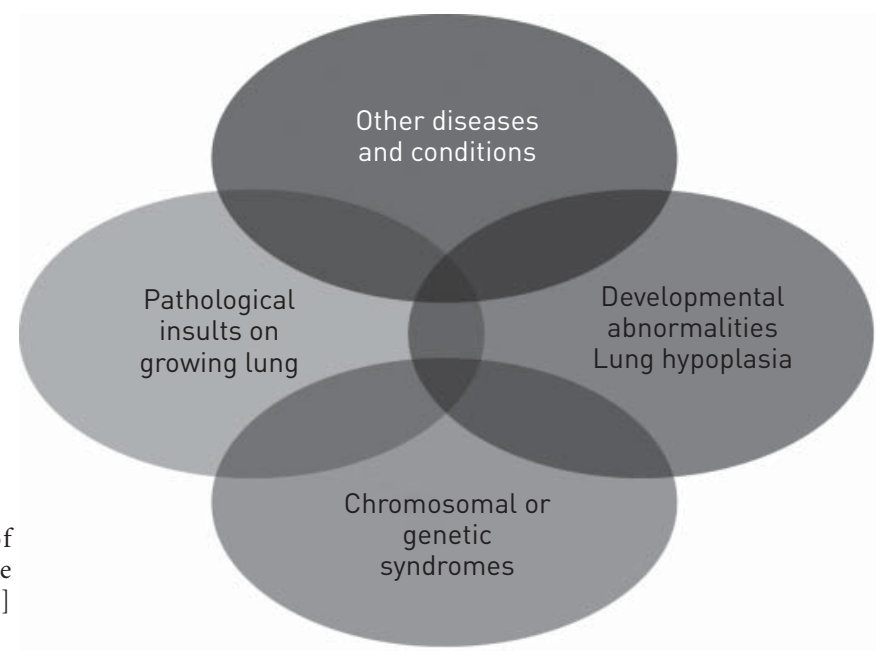


Paediatric PAH seems to be associated with a similar life expectancy to that seen in adults [14]. The REVEAL (Registry to Evaluate Early and Long-Term Pulmonary Arterial Hypertension Disease Management) registry reported mean \pm SD 1-, 3- and 5-year estimated survival rates of $96 \pm 4 \%, 84 \pm 5 \%$ and $74 \pm 6 \%$, respectively, for childhood-onset $\mathrm{PAH}[3,15]$. However, most of these patients were prevalent, and survival for incident patients may indeed be worse due to survival bias. Although overall survival has improved in recent years for certain patient populations, such as those with repaired CHD and pulmonary hypertensive vascular disease, poor prognosis remains $[4,16,17]$.

\section{Prognostic factors in paediatric PAH patients}

A list of currently known prognostic risk factors is included in table 1. Some of these factors mirror those seen in adults, such as right ventricular failure or progression to World Health Organization (WHO) functional class III or IV [18]. Specific haemodynamic variables, such as systemic cardiac index $<2.5 \mathrm{~L} \cdot \mathrm{min}^{-1} \cdot \mathrm{m}^{-2}$, mean pulmonary arterial pressure/mean systemic arterial pressure $>0.75 \mathrm{mmHg}$, right atrial pressure $>10 \mathrm{mmHg}$ or PVR index $>20 \mathrm{Wood}$ Unit $\cdot \mathrm{m}^{-2}$, are associated with worse prognosis in paediatric patients [18]. There are conflicting reports regarding the predictive value of the 6 -min walk test in children with PAH $[7,16,19,20]$. Emerging evidence indicates that the serum level of B-type natriuretic peptide or N-terminal pro-brain natriuretic peptide (NT-proBNP) is associated with exercise capacity [21-23] and predicts prognosis [24]. Specific to paediatric patients, failure to thrive has been reported to be associated with a higher risk of death [7]. A prospective follow-up study of 66 treatment-naïve paediatric patients with PAH examined the value of various clinical, biochemical and echocardiographic measurements in predicting transplant-free survival. Treatment-induced changes in WHO functional class, NT-proBNP and tricuspid annular plane systolic excursion (TAPSE) were associated with improved survival [25].

\section{Challenges associated with diagnosis of PAH in children}

Results from the TOPP registry revealed that a full diagnostic work-up is rarely performed in paediatric patients with suspected pulmonary hypertension [5], possibly due to the difficulty of ensuring cooperation in paediatric patients or the associated risks of certain procedures (such as heart catheterisation, magnetic resonance imaging or lung biopsy) [2].

Diagnostic work-up (observed symptoms and diagnostic testing) of paediatric PAH is, in principle, similar to that utilised in the adult population [18]. Right heart catheterisation is essential for PAH diagnosis (using the same haemodynamic definition as in adults). This may include acute vasoreactivity testing, which has been shown in some studies to be associated with higher response rates in paediatric patients than adults [26]. This finding has not been reproduced consistently, however, and may be an artefact of different criteria used in different studies [7, 27]. While important, diagnostic heart catheterisation may be more frequently associated with more complications in children than in adults and as such requires specific expertise [2].

\section{TABLE 1 Determinants of risk in paediatric pulmonary arterial hypertension}

$\begin{array}{lll}\text { Determinants of risk Lower risk Higher risk } & \text { Lo }\end{array}$

Clinical evidence of RV failure
Progression of symptoms
Syncope
Growth
WHO functional class
BNP/NT-proBNP
Echocardiography

Haemodynamics

$\begin{array}{cc}\text { No } & \text { Yes } \\ \text { No } & \text { Yes } \\ \text { No } & \text { Yes } \\ \text { I, II } & \text { Failure to thrive } \\ \text { III, IV } \\ \text { Minimally elevated } & \text { Significantly elevated, increasing } \\ & \text { Severe RV enlargement/dysfunction } \\ \text { Systemic Cl }>3.0 \mathrm{~L} \cdot \mathrm{min}^{-1} \cdot \mathrm{m}^{-2} & \text { Pericardial effusion } \\ \mathrm{mPAP} / \mathrm{mSAP}<0.75 \mathrm{mmHg}^{-2} & \mathrm{mPAP} / \mathrm{mSAP}>0.75 \mathrm{mmHg}^{-2} \\ \text { Acute vasoreactivity } & \mathrm{RAP}>10 \mathrm{~mm} \mathrm{Hg} \\ & \mathrm{PVRI}>20 \mathrm{WU} \cdot \mathrm{m}^{-2}\end{array}$

RV: right ventricular; WHO: World Health Organization; BNP: B-type natriuretic peptide; NT-proBNP: Nterminal pro-BNP; $\mathrm{Cl}$ : cardiac index; mPAP: mean pulmonary artery pressure; mSAP: mean systemic artery pressure; RAP: right atrial pressure; PVRI: pulmonary vascular resistance index; WU: Wood unit. Reproduced from [18] with permission from the publisher. 
Echocardiography is an important noninvasive tool for the diagnosis of $\mathrm{PAH}$, since it can indicate elevated pulmonary artery pressure [25, 28, 29]. However, echocardiography is not a substitute for right heart catheterisation since it cannot properly measure pulmonary artery pressure, PVR and pulmonary capillary wedge pressure $[29,30]$. Therefore, right heart catheterisation is still required to confirm the diagnosis of $\mathrm{PAH}$. Echocardiography is also useful for patient follow-up once therapy has been initiated [28], and is used for both assessing and predicting outcomes in paediatric patients through, for example, the measurement of right/left ventricle ratio and right ventricle stroke work $[29,31]$. Changes in TAPSE are also of prognostic value [25].

\section{Evidence for treatment of paediatric PAH}

Compared to the evidence base in adults, the evidence base for paediatric $\mathrm{PAH}$ therapy is currently limited [18]. As a result, the recently proposed IPAH/heritable PAH treatment algorithm (fig. 2) is primarily based on expert opinion [18]. As a first step, referral to an expert centre is recommended. As disease awareness increases, there is a possibility that children with suspected PAH may, in some cases, be started on initial treatment by paediatric cardiologists or respirologists in place of immediate referral to a pulmonary hypertension centre. This practice may impact on the diagnosis of rarer subtypes of pulmonary (arterial) hypertension, restrict or prevent inclusion in national disease registries and, in some cases, delay timely treatment intensification. The decision to use supportive therapies such as warfarin as an anticoagulant, diuretics for symptomatic management of right ventricle volume overload, oxygen to maintain $>90 \%$ oxygen saturation or digoxin to increase cardiac output, should be based on an individual patient's requirements, but specific studies to support this remain scarce [18]. Following a full diagnostic work-up, patients who may be eligible for long-term, oral calcium channel blocker (CCB) therapy should undergo acute vasoreactivity testing and, in the presence of a positive response, may commence CCB therapy [26]. For non-vasoreactive patients or those failing to respond to CCB therapy, the approach to treatment is currently based on risk of death (table 1), with lower risk patients typically commencing oral monotherapy. Data regarding the efficacy and safety of PAH-targeted monotherapy in children are limited to endothelin receptor agonists [32,33], intravenous or subcutaneous prostanoids [34], and the phosphodiesterase type-5 (PDE5) inhibitors sildenafil $[35,36]$ and tadalafil [37]. Sildenafil is currently the only approved treatment in Europe for PAH in children, based on data from the 16-week STARTS-1 (Sildenafil in Treatment-Naïve Children, Aged 1-17 Years, with Pulmonary Arterial Hypertension) trial, reporting improvements in peak oxygen consumption (primary end-point) at higher sildenafil doses [36]. Unexplained increased mortality was observed in patients receiving higher doses of sildenafil during the trial extension STARTS-2 [35]. As a result this treatment is not currently approved for paediatric patients with PAH in the USA.

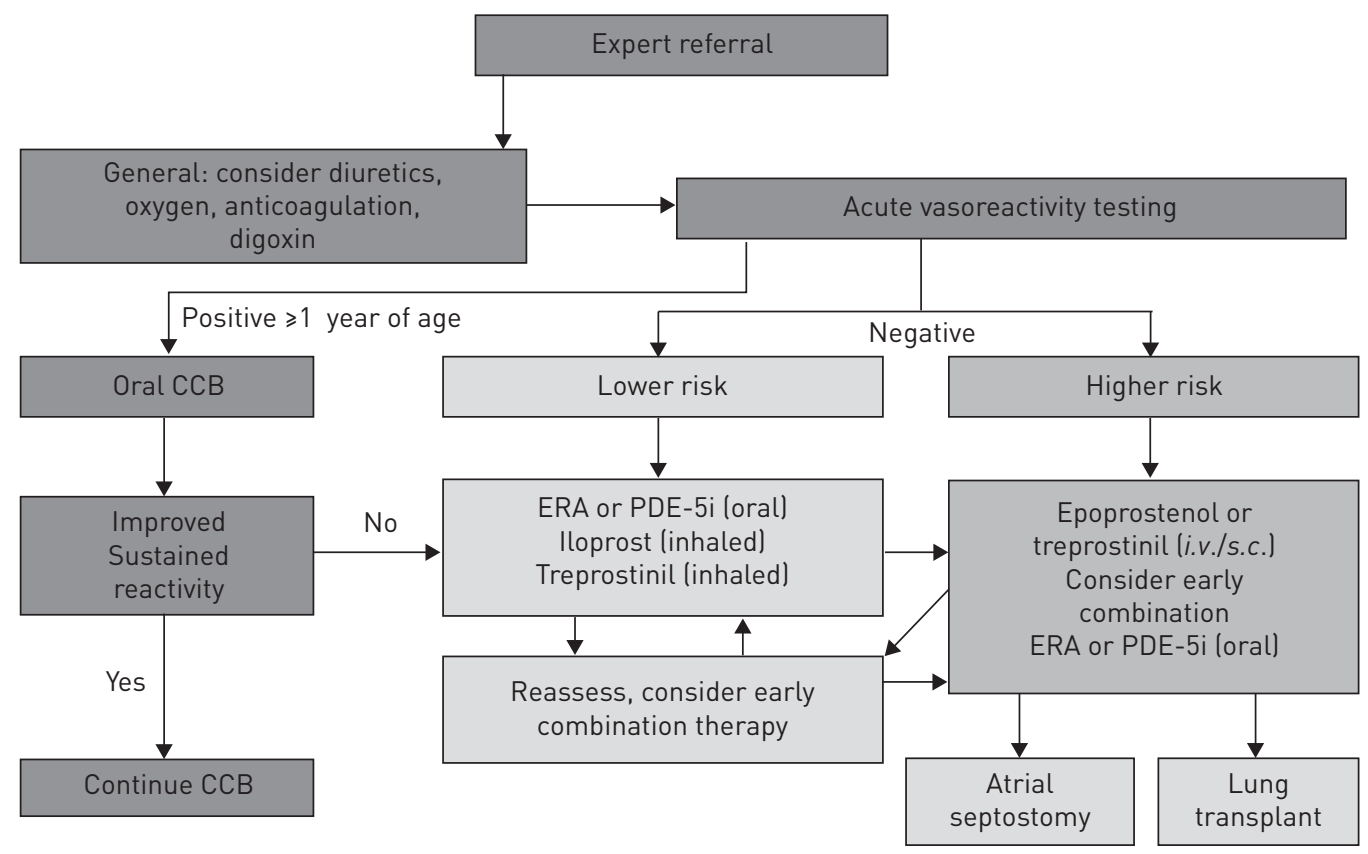

FIGURE 2 The recently proposed paediatric idiopathic/hereditable pulmonary arterial hypertension treatment algorithm. In Europe, the use of all agents is considered off-label in children, aside from sildenafil. CCB: calcium channel blocker; ERA: endothelin receptor antagonist; PDE-5i: phosphodiesterase type-5 inhibitor. Reproduced from [18] with permission from the publisher. 
The investigation of treatment in paediatric PAH has highlighted the potential for differences to adults in pharmacokinetics and dose response [32].

Higher risk paediatric patients may be considered for combination therapy. Registry data suggest a potential clinical benefit for combination therapy compared with monotherapy in paediatric PAH [38]. The addition of an inhaled prostacyclin to endothelin receptor agonists or PDE-5 inhibitors may be beneficial; however, this is based on evidence from a retrospective study of 22 patients across five pulmonary hypertension clinics [39]. In cases of paediatric patients at higher risk of deterioration, switching to i.v. prostacyclin should be considered $[34,40]$. For patients in WHO functional class IV, patients should be started on i.v. prostacyclin, potentially in combination with oral endothelin receptor agonists or PDE-5 inhibitors.

In cases where drug therapy does not prevent worsening of $\mathrm{PAH}$, surgical alternatives may be utilised. Atrial septostomy may be considered as an alternative to transplantation. The Potts shunt has also been shown to improve functional status and survival in severely ill children [41].

It is clear that while improvements have been and continue to be made in our understanding of treatments for paediatric PAH, these are currently primarily based on registry data, complemented by individual clinical experience, short-term trials and open-label studies. In order to make progress towards safe, effective therapies in paediatric $\mathrm{PAH}$, it is important to understand the difficulties associated with collecting clinical evidence in this population.

\section{Challenges of clinical trials in paediatric PAH}

The relative lack of evidence for treatments in paediatric $\mathrm{PAH}$, compared to the adult population, is fundamentally linked to challenges in designing and conducting clinical trials that cover a wide range of ages and stages of development. Normal physical development throughout childhood and adolescence leads to changes in hormone levels and metabolism, resulting in potential changes in pharmacokinetic and/or pharmacodynamic parameters [21]. As a result, there is a need for trials that investigate the long-term safety of PAH-targeted treatments, particularly considering the example of unanticipated dose responses observed previously [35]. The particular issues associated with such trials can be broadly grouped into regulatory requirements, defining a homogeneous study population and lack of validated end-points in this population.

\section{Regulatory requirements}

The European Medicines Agency (EMA) and the US Food and Drug Administration have issued guidelines for the conduct of randomised controlled trials (RCTs) in paediatric PAH. EMA guidelines require strong pharmacokinetic/pharmacodynamic and safety data to be provided; consequently, a number of pharmacokinetic and safety studies have been initiated, but no RCTs of efficacy have been conducted. The EMA advocates the extrapolation of results from adults to children with PAH if efficacy is proved in adults and the disease in children is comparable; however, a dose-finding study is required and a stepwise approach recommended, beginning with older children in the first instance. The Food and Drug Administration requires a powered RCT to be conducted in the paediatric pulmonary hypertension setting with a significant clinical end-point for evidence of efficacy. Therefore, to design a feasible RCT, surrogate end-points are needed. Haemodynamic parameters are currently not accepted as a surrogate due to the potential risks associated with cardiac catheterisation in children. 6-min walking distance (6MWD) has been accepted, but it is difficult to measure consistently, particularly in younger children. Noninvasive endpoints that require further evaluation in paediatric patients include echocardiographic parameters such as the systolic to diastolic duration ratio, tissue Doppler indices, eccentricity index and TAPSE [18]. TAPSE improvement has recently been reported as being a valuable goal to reach for children with PAH [25]. Further work is needed to validate echocardiographic parameters as potential treatment goals for paediatric patients with PAH. Other end-points, such as time to clinical worsening, may be considered; however, this would require a significant number of patients to be recruited, which remains a challenge in the paediatric population. In addition, a pure morbidity/mortality study, such as recent adult studies, would be ideal but again they face the problems of recruitment and length of study. Taken together, these issues highlight the difficult task of balancing expectations regarding recruitment and retention, with the identification of clinically meaningful trial outcomes.

\section{Recruitment and retention}

RCTs in paediatric PAH face ethical challenges such as the feasibility of a placebo-controlled trial in patients who may benefit from additional treatments that are often used off-label in clinical practice [42]. In addition to the lack of validated clinical trial end-points, which is a specific consideration in paediatric PAH [42], other challenges in designing rigorous trials include duration (given the wide age range and survival rates of paediatric patients) and, importantly, identifying patients on similar background monotherapy. Recruitment is exacerbated by disease rarity and the heterogeneity of PAH in infants and children; in addition to a tendency 
to focus on patients without shunts, which significantly reduces the number of eligible patients. This problem is highlighted by registry findings. Of the 600 patients in the TOPP registry, only $3 \%$ were included in clinical trials [5].

\section{Clinical trial end-points}

In order to design meaningful clinical trials for children with $\mathrm{PAH}$, a consensus must be reached on clinical end-points or treatment goals that are applicable across paediatric age groups and can be clearly linked to clinical outcomes. Patient registries that specifically aim to assess the appropriateness of treatment variables, via a standardised collection process, will provide further clarity on such end-points [25].

Adult RCTs in PAH are showing a shift towards more long-term designs, focusing on mortality and morbidity end-points rather than changes in the 6MWD. Such trial designs may not be possible in the paediatric setting due to the low numbers of patients that could potentially be recruited, particularly considering the power required to examine relatively infrequent events such as mortality. This limited patient pool is further reduced when considering longer term studies, as paediatric patients in clinical trials may not remain on a single therapy for a prolonged period of time due to advances in standard of care. In spite of this challenge, long-term safety follow-up is of importance in determining the effects of PAHspecific therapies on growth, puberty and fertility in paediatric patients [21].

Challenges in exercise capacity or cardiopulmonary exercise testing in the younger or uncooperative child remain [21], while the performance of echocardiography has not yet been sufficiently studied in children or adults. In addition, it may be difficult to reproduce findings in multicentre trials accurately. Cardiac catheterisation, while a more reliable means of assessing disease severity in children, is invasive, often requires sedation or general anaesthesia and carries a higher risk of complications in children than in adults [43].

Biomarkers offer the potential of a reliable, age-independent indicator of disease severity, with the use of NT-proBNP currently seen as the most promising tool for potential use as a treatment goal in paediatric PAH [22-25]. Further studies will be required to ascertain the true predictive value of these and other biomarkers in individual patients [21]. Based on current evidence, it appears likely that the use of a combined end-point may be the most appropriate strategy for studies in the paediatric population.

\section{Conclusion}

In spite of the challenges inherent to the treatment of paediatric PAH patients and the limited evidence available, physicians, pharmaceutical companies and regulators continue to take steps to improve treatment availability. However, there is still a need for a suitable way to build on the current body of evidence in a larger paediatric patient population and thereby support the development of treatment strategies for paediatric $\mathrm{PAH}$.

\section{Acknowledgements}

Medical writing support was provided by Nason Ma'ani (apothecom scopemedical ltd, Sevenoaks, UK), which was funded by Actelion Pharmaceuticals Ltd (Allschwil, Switzerland).

\section{References}

1 Humbert M, Morrell NW, Archer SL, et al. Cellular and molecular pathobiology of pulmonary arterial hypertension. J Am Coll Cardiol 2004; 43: Suppl. 12, 13S-24S

2 Beghetti M, Berger RM, Schulze-Neick I, et al. Diagnostic evaluation of paediatric pulmonary hypertension in current clinical practice. Eur Respir J 2013; 42: 689-700.

3 McGoon MD, Miller DP. REVEAL: a contemporary US pulmonary arterial hypertension registry. Eur Respir Rev 2012; 21: 8-18.

4 van Loon RL, Roofthooft MT, Hillege HL, et al. Pediatric pulmonary hypertension in the Netherlands: epidemiology and characterization during the period 1991 to 2005. Circulation 2011; 124: 1755-1764.

5 Berger RM, Beghetti M, Humpl T, et al. Clinical features of paediatric pulmonary hypertension: a registry study. Lancet 2012; 379: 537-546.

6 van Loon RL, Roofthooft MT, Delhaas T, et al. Outcome of pediatric patients with pulmonary arterial hypertension in the era of new medical therapies. Am J Cardiol 2010; 106: 117-124.

7 Moledina S, Hislop AA, Foster H, et al. Childhood idiopathic pulmonary arterial hypertension: a national cohort study. Heart 2010; 96: 1401-1406.

8 Farrow KN, Fliman P, Steinhorn RH. The diseases treated with ECMO: focus on PPHN. Semin Perinatol 2005; 29: $8-14$.

9 del Cerro MJ, Abman S, Diaz G, et al. A consensus approach to the classification of pediatric pulmonary hypertensive vascular disease: report from the PVRI Pediatric Taskforce, Panama 2011. Pulm Circ 2011; 1: 286-298.

10 Simonneau G, Gatzoulis M, Adatia I, et al. Updated clinical classification of pulmonary hypertension. J Am Coll Cardiol 2013; 62: Suppl. 25, D34-D41

11 Humbert M, Sitbon O, Chaouat A, et al. Pulmonary arterial hypertension in France: results from a national registry. Am J Respir Crit Care Med 2006; 173: 1023-1030. 
12 Badesch DB, Raskob GE, Elliott CG, et al. Pulmonary arterial hypertension: baseline characteristics from the REVEAL Registry. Chest 2010; 137: 376-387.

13 Schulze-Neick I, Beghetti M. Issues related to the management and therapy of paediatric pulmonary hypertension. Eur Respir Rev 2010; 19: 331-339.

14 Sandoval J, Bauerle O, Gomez A, et al. Primary pulmonary hypertension in children: clinical characterization and survival. J Am Coll Cardiol 1995; 25: 466-474.

15 Barst RJ, McGoon MD, Elliott CG, et al. Survival in childhood pulmonary arterial hypertension: insights from the registry to evaluate early and long-term pulmonary arterial hypertension disease management. Circulation 2012; 125: $113-122$.

16 Haworth SG, Hislop AA. Treatment and survival in children with pulmonary arterial hypertension: the UK Pulmonary Hypertension Service for Children 2001-2006. Heart 2009; 95: 312-317.

17 Manes A, Palazzini M, Leci E, et al. Current era survival of patients with pulmonary arterial hypertension associated with congenital heart disease: a comparison between clinical subgroups. Eur Heart J 2014; 35: 716-724.

18 Ivy DD, Abman SH, Barst RJ, et al. Pediatric pulmonary hypertension. J Am Coll Cardiol 2013; 62: Suppl. 25, D117-D126

19 Lammers AE, Diller GP, Odendaal D, et al. Comparison of 6-min walk test distance and cardiopulmonary exercise test performance in children with pulmonary hypertension. Arch Dis Child 2011; 96: 141-147.

20 Lammers AE, Munnery E, Hislop AA, et al. Heart rate variability predicts outcome in children with pulmonary arterial hypertension. Int J Cardiol 2010; 142: 159-165.

21 Adatia I, Haworth SG, Wegner M, et al. Clinical trials in neonates and children: report of the pulmonary hypertension academic research consortium pediatric advisory committee. Pulm Circ 2013; 3: 252-266.

22 Lammers AE, Hislop AA, Haworth SG. Prognostic value of B-type natriuretic peptide in children with pulmonary hypertension. Int J Cardiol 2009; 135: 21-26.

23 Takatsuki S, Wagner BD, Ivy DD. B-type natriuretic peptide and amino-terminal pro-B-type natriuretic peptide in pediatric patients with pulmonary arterial hypertension. Congenit Heart Dis 2012; 7: 259-267.

24 Van Albada ME, Loot FG, Fokkema R, et al. Biological serum markers in the management of pediatric pulmonary arterial hypertension. Pediatr Res 2008; 63: 321-327.

25 Ploegstra M-J, Douwes JM, Roofthooft MTR, et al. Identification of treatment goals in paediatric pulmonary arterial hypertension. Eur Respir J 2014; 44: 1616-1626.

26 Barst RJ, Maislin G, Fishman AP. Vasodilator therapy for primary pulmonary hypertension in children. Circulation 1999; 99: 1197-1208.

27 Douwes JM, van Loon RL, Hoendermis ES, et al. Acute pulmonary vasodilator response in paediatric and adult pulmonary arterial hypertension: occurrence and prognostic value when comparing three response criteria. Eur Heart J 2011; 32: 3137-3146.

28 Colvin KL, Dufva MJ, Delaney RP, et al. Biomarkers for pediatric pulmonary arterial hypertension - a call to collaborate. Front Pediatr 2014; $2: 7$.

29 Jone PN, Hinzman J, Wagner BD, et al. Right ventricular to left ventricular diameter ratio at end-systole in evaluating outcomes in children with pulmonary hypertension. J Am Soc Echocardiogr 2014; 27: 172-178.

30 Kirkpatrick EC. Echocardiography in pediatric pulmonary hypertension. Paediatr Respir Rev 2013; 14: 157-164.

31 Di Maria JM, Younoszai AK, Mertens L, et al. RV stroke work in children with pulmonary arterial hypertension: estimation based on invasive haemodynamic assessment and correlation with outcomes. Heart 2014; 100: 1342-1347.

32 Beghetti M, Haworth SG, Bonnet D, et al. Pharmacokinetic and clinical profile of a novel formulation of bosentan in children with pulmonary arterial hypertension: the FUTURE-1 study. Br J Clin Pharmacol 2009; 68: 948-955.

33 Takatsuki S, Rosenzweig EB, Zuckerman W, et al. Clinical safety, pharmacokinetics, and efficacy of ambrisentan therapy in children with pulmonary arterial hypertension. Pediatric Pulmonol 2013; 48: 27-34.

34 Siehr SL, Ivy DD, Miller-Reed K, et al. Children with pulmonary arterial hypertension and prostanoid therapy: long-term hemodynamics. J Heart Lung Transplant 2013; 32: 546-552.

35 Barst RJ, Beghetti M, Pulido T, et al. STARTS-2: long-term survival with oral sildenafil monotherapy in treatmentnaïve pediatric pulmonary arterial hypertension. Circulation 2014; 129: 1914-1923.

36 Barst RJ, Ivy DD, Gaitan G, et al. A randomized, double-blind, placebo-controlled, dose-ranging study of oral sildenafil citrate in treatment-naive children with pulmonary arterial hypertension. Circulation 2012; 125: 324-334.

37 Takatsuki S, Calderbank M, Ivy DD. Initial experience with tadalafil in pediatric pulmonary arterial hypertension. Pediatr Cardiol 2012; 33: 683-688.

38 Zijlstra WM, Douwes JM, Rosenzweig EB, et al. Survival differences in pediatric pulmonary arterial hypertension: clues to a better understanding of outcome and optimal treatment strategies. J Am Coll Cardiol 2014; 63: 2159-2169.

39 Ivy DD, Doran AK, Smith KJ, et al. Short- and long-term effects of inhaled iloprost therapy in children with pulmonary arterial hypertension. J Am Coll Cardiol 2008; 51: 161-169.

40 Levy M, Celermajer DS, Bourges-Petit E, et al. Add-on therapy with subcutaneous treprostinil for refractory pediatric pulmonary hypertension. J Pediatr 2011; 158: 584-588.

41 Baruteau AE, Serraf A, Lévy M, et al. Potts shunt in children with idiopathic pulmonary arterial hypertension: longterm results. Ann Thorac Surg 2012; 94: 817-824.

42 Berger RM. Pulmonary hypertension: smaller kids, smaller steps. Lancet Respir Med 2014; 2: 348-350.

43 Taylor CJ, Derrick G, McEwan A, et al. Risk of cardiac catheterization under anaesthesia in children with pulmonary hypertension. Br J Anaesth 2007; 98: 657-661. 